\title{
Transcriptomic differential IncRNA expression is involved in neuropathic pain in rat dorsal root ganglion after spared sciatic nerve injury
}

\author{
P. Mao ${ }^{1}$, C.R. Li ${ }^{1}$, S.Z. Zhang ${ }^{2}$, Y. Zhang ${ }^{1}$, B.T. Liu ${ }^{1}$ and B.F. Fan ${ }^{1}$ \\ ${ }^{1}$ Department of Pain Medicine, China-Japan Friendship Hospital, Beijing, China \\ ${ }^{2}$ State Key Laboratory of Toxicology and Medical Countermeasures, Department of Biochemical Pharmacology, \\ Beijing Institute of Pharmacology and Toxicology, Beijing, China
}

\begin{abstract}
Dorsal root ganglia (DRG) neurons regenerate spontaneously after traumatic or surgical injury. Long noncoding RNAs (IncRNAs) are involved in various biological regulation processes. Conditions of IncRNAs in DRG neuron injury deserve to be further investigated. Transcriptomic analysis was performed by high-throughput Illumina HiSeq2500 sequencing to profile the differential genes in L4-L6 DRGs following rat sciatic nerve tying. A total of 1,228 genes were up-regulated and 1,415 down-regulated. By comparing to rat IncRNA database, 86 known and 26 novel IncRNA genes were found to be differential. The 86 known IncRNA genes modulated 866 target genes subject to gene ontology (GO) and KEGG enrichment analysis. The genes involved in the neurotransmitter status of neurons were downregulated and those involved in a neuronal regeneration were upregulated. Known IncRNA gene rno-Cntnap2 was downregulated. There were 13 credible GO terms for the rno-Cntnap2 gene, which had a putative function in cell component of voltage-gated potassium channel complex on the cell surface for neurites. In 26 novel IncRNA genes, 4 were related to 21 mRNA genes. A novel IncRNA gene AC111653.1 improved rno-Hypm synthesizing huntingtin during sciatic nerve regeneration. Real time qPCR results attested the down-regulation of rno-Cntnap IncRNA gene and the upregulation of $A C 111653.1$ IncRNA gene. A total of 26 novel IncRNAs were found. Known IncRNA gene rno-Cntnap2 and novel IncRNA AC111653.1 were involved in neuropathic pain of DRGs after spared sciatic nerve injury. They contributed to peripheral nerve regeneration via the putative mechanisms.
\end{abstract}

Key words: Spared nerve injury; Neuropathic pain; Long noncoding RNA; Transcriptome; Hypm gene

\section{Introduction}

After traumatic damage, the peripheral nervous system can regenerate spontaneously by activating the inherent growth ability of neurons, while the central nervous system cannot do so $(1,2)$. The sciatic nerve is commonly used as a model to study peripheral nerve regeneration. It includes a complex bunch of motor and sensory axons, in which the sensory neurons are situated in the L4-L6 dorsal root ganglion (DRG) $(3,4)$. After sciatic nerve damage, the damaged neurons initiate a regeneration process but cease having the neurotransmitter status $(3,5)$. Axon regeneration and pathfinding after damage involves a complex mechanism involving axon cross-talk with neurogliocytes, nerve growth factors, neurotrophic factors, and receptors $(6,7)$.

Neuropathic pain after traumatic or surgical nerve injury challenges doctors and patients and regulatory noncoding RNAs (ncRNAs) are key molecules for understanding and treating this pain $(8,9)$. Regulatory ncRNAs are transcribed from protein noncoding genes to interfere in gene expression and they include, but are not limited to, miRNAs (21-24 nt), siRNAs (21-25 bp), piRNAs (26-31 nt), and long noncoding RNAs (IncRNAs, 200 bp to more than 100,000 bp) $(8,10,11)$. The role of miRNAs, IncRNAs, and piRNAs in neuropathic pain after nerve injury has been reviewed by Bali and Kuner (8). The role and regulatory mechanisms of IncRNAs in vertebrate central nervous system and human nervous system diseases have been reported in the literature $(8,12-15)$.

In peripheral nerve injury, IncRNAs play an important role in stress responses, plasticity, and axonal outgrowth of DRG neurons $(8,16-21)$. Yu et al. (16) investigated the IncRNA transcriptome of DRG neurons after sciatic nerve injury in rat models and found that IncRNAs modulate DRG neurons responses to ligation stimuli. Zhao et al. (17) identified the modulating effect of the IncRNA (Kcna2 antisense RNA) on a voltage-dependent potassium channel mRNA

Correspondence: B.F. Fan: <doctormaopeng@163.com> 
Kcna2 in primary afferent neurons. Yao et al. (18) reported that the IncRNA uc. 217 modulates neurite outgrowth of DRG neurons after sciatic nerve injury. IncRNA NONRATT 021972 and IncRNA uc.48 modulate neuropathic pain mediated by the $\mathrm{P} 2 \mathrm{X}(3 / 7)$ purinergic receptors (the cationpermeable ATP-binding ligand-gated ion channels) in the DRG neurons in diabetic rat models, and siRNA therapy alleviate the pain significantly (19-23). However, all these studies are based on microarray analysis (24). A sequencing study to elucidate vital roles of IncRNAs in peripheral nerve pathology will help to understand neuropathic pain.

In this study, we described the IncRNAs expression and $\mathrm{mRNA}$ expression in DRGs during nerve regeneration by a transcriptome-level deep sequencing. The results reveal a novel layer of regulation of the inherent growth ability of neurons by IncRNAs.

\section{Material and Methods}

\section{Animal surgery and sample preparation}

Six male Sprague-Dawley rats $(180-220 \mathrm{~g})$ were housed in large cages with sawdust bedding at $25^{\circ} \mathrm{C}$ in $12 \mathrm{~h} / 12 \mathrm{~h}$ dark/light cycle and allowed free access to food and water in the Animal Center of Beijing China-Japan Friendship Hospital. Rats were randomly divided into test group and sham-operation group, three in each group. Surgery was performed as described in the literature with modifications (16). Briefly, rats were anesthetized by intraperitoneal injection of $10 \mathrm{wt} \%$ chloral hydrate $(3 \mathrm{~mL} / \mathrm{kg}$, Tianjin Fuchen Chemical Reagent Factory, China). The sciatic nerves were exposed and lifted through an incision on the right lateral thigh. Sciatic nerve segments were tied at the site proximal to the bifurcation of tibial and common peroneal nerves. Rats in the sham-operation group only had the sciatic nerves exposed without tying. L4-L6 DRGs were harvested from each animal a week later. All animal experiments were performed in accordance with institutional animal welfare and care guidelines and approved by the Animal Ethics Committee of Beijing China-Japan Friendship Hospital.

\section{RNA isolation, cDNA library preparation, and sequencing}

Total RNAs were extracted from the L4-L6 DRG tissues using Trizol reagent according to the instructions of the manufacturer (Invitrogen, USA). RNAs were cleaned, including a DNase I digestion step, using RNeasy spin columns (Qiagen, USA). RNA integrity was detected by agarose gel electrophoresis and RNA was quantified using Nanodrop2000 (Bio-Rad, USA). After rRNA was removed, RNA was interrupted into short fragments by adding fragmentation buffer. These short RNA fragments were used as templates to synthesize the firststrand cDNA using FastQuant RT Kit (with gDNAase) (Tiangen, China). Then, double-strand cDNA was obtained.
The cDNA products were purified with QiaQuick PCR extraction kit (Qiagen, Germany) and the purified cDNA were dissolved in EB buffer, followed by end reparation and $\operatorname{poly}(A)$ addition. The cDNA fragments were connected to sequencing adaptors. The cDNA fragments at 150-200 bp in size were separated on gel-electrophoresis and were used as the templates for PCR amplification. Two cDNA libraries for the test and sham-operation groups were sequenced using the Illumina HiSeq2500 at Beijing Ori-Gene Science and Technology Co., LTD (China).

\section{Data processing}

Raw images generated by sequencers were converted by Illumina software (USA) to nucleotide sequences, called raw reads. FastQC software package (USA) was used to generate clean reads by removing adaptor reads, low quality reads (QC30), sequences containing fuzzy $N$ bases and sequences less than $60 \mathrm{bp}$. All the following analyses were based on clean reads. The clean reads were mapped to the genome using Tophat2 software package (USA). RSeQC software package with all default parameters (USA) was used to detect the splice junction sites for evaluating the saturation of sequencing.

\section{Differential expression and enrichment}

The RPKM method (reads per kilobase per million mapped reads) was used to calculate the differential expression level using the Cufflinks software package (USA). Cufflinks Cuffdiff was used to screen the differential expression genes (DEGs). The criterion to identify the DEGs between two groups was as follows: sum of mapping reads of two samples $\geqslant 10$; |log2RPKM fold change $>1$; $P$ value was corrected by false discovery rate (FDR) to get a $Q$-value; both $P \leqslant 0.05$ and $Q \leqslant 0.05$ were required to determine the significance of differential expression. In order to get biological functions, DEGs were subjected to gene ontology (GO) and Kyoto Encyclopedia of Genes and Genomes (KEGG) enrichment analyses. R package was used for the analysis. GO terms of DEGs were compared with the genome background and the corrected $\mathrm{P}$ value (FDR correction) < 0.05 was set to judge the significantly enriched $\mathrm{GO}$ terms. For the KEGG pathway enrichment analysis, $\mathrm{P}$ value $<$ 0.05 was the threshold.

\section{Known and novel IncRNA}

The RPKM method was used to calculate the known expression level using the Cufflinks software package. Because IncRNAs have no conservation between species, we used rat IncRNA database for annotating known IncRNA in the transcript obtained from sequencing. New transcripts with opening read frame features were aligned with known protein database with CPC scoring to predict either coding or non-coding RNA. The predicted noncoding RNA is a novel IncRNA. 


\section{Coexpression network and target gene enrichment analysis}

By comparing the differential gene lists, we obtained gene pairs of novel IncRNA and differential mRNA. FPKM values for each pair were used to calculate the Pearson correlation, where we chose significantly correlated gene pairs (correlation coefficient $>0.995$ or $<-0.995, \mathrm{P}<0.05$ as threshold) to build a coexpression network using Cytoscape software package (USA). GO and KEGG enrichment analyses were performed as described above but the corrected $P$ value threshold was set to $<0.1$. The correlated mRNA genes that coexpressed with IncRNAs served as the candidate target genes for IncRNAs.

\section{Real-time qPCR quantification}

To quantify IncRNA and target mRNAs, real-time qPCR were performed on representative rno-Cntnap2201 IncRNA gene, rno-Fam171b mRNA gene, rno-Hebp2 mRNA gene, rno-Gde1 mRNA gene, rno-AC111653.1 IncRNA gene, and rno-Hypm mRNA gene in the shamoperation and test DRG groups. Briefly, the first-strand cDNA was synthesized using FastQuant RT Kit (with gDNAase) (Tiangen). Then, double-strand cDNA was synthesized. Real time qPCR was performed on a Roche LightCycler $^{\mathrm{R}} 96$ fluorescence quantifying PCR machine. Primer sequences are listed in Table 1.

The reaction system included $1 \mu \mathrm{L}$ of cDNA, $10 \mu \mathrm{L}$ of RealStar Green Mixture $(2 \times), 0.6 \mu \mathrm{L}$ of primers, and filled up to $20 \mu \mathrm{L}$ with PCR-grade water. The PCR program included a pre-denaturation at $95^{\circ} \mathrm{C}$ for $5 \mathrm{~min}, 40$ cycles $\left(95^{\circ} \mathrm{C}\right.$ for $15 \mathrm{~s}, 60^{\circ} \mathrm{C}$ for $20 \mathrm{~s}, 72^{\circ} \mathrm{C}$ for $\left.15 \mathrm{~s}\right)$ for amplification, and a default condition for dissociation. The cycle threshold (Ct) values were obtained. Relative interest/ reference mRNA expression was calculated by the formula: $2-\Delta \mathrm{Ct}$ (interest-reference). rno-GAPDH was used as inner reference.

Table 1. Primer sequences.

\begin{tabular}{lc}
\hline Gene name & Sequence $\left(5^{\prime} \rightarrow 3^{\prime}\right)$ \\
\hline rno-Cntnap2-201_F & gcacctaccacaccaacga \\
rno-Cntnap2-201_R & tttgctctcgtcaatggtctct \\
rno-Fam171b_5267F & aggagttctgcttgctctgg \\
rno-Fam171b_5460R & tccacacacaaccaagggta \\
rno-Hebp2_415F & agatccgacactacggacca \\
rno-Hebp2_662R & cctgggtggatcatgttgct \\
rno-Gde1_20F & acgggtctggccgattatgt \\
rno-Gde1_586R & gcactctgtaactgcttcct \\
rno-GAPDH_1096F & gcccagcaaggatactgaga \\
rno-GAPDH_1252R & ggtattcgagagaagggaggg \\
rno-AC111653.1_201F & agctacagtcatggaaacaccc \\
rno-AC111653.1_201R & agatagcctcagcttgctcact \\
rno-Hypm_71F & cgacatgatg gtttgatggt \\
rno-Hypm_251R & ccatgcttga ttaccttacc \\
\hline
\end{tabular}

\section{Cell assays}

Wistar rat DRG cells were isolated and cultured as reported in the literature (25). Cell assays were performed as reported in the literature with a minor modification (26). Briefly, one-day-old Wistar rat DRG cells were isolated and cultured in 95\% Eagle's DMEM feeding medium with $600 \mathrm{mg} / \mathrm{mL}$ glucose, $10 \%$ fetal bovine serum, $5 \%$ horse serum, $20 \mathrm{ng} / \mathrm{mL}$ nerve growth factors, and $1 \mu \mathrm{g} / 100 \mathrm{~mL}$ neurotrophins (25). For RNA silencing, siRNA sequences targeting Inc-AC111653.1 were designed and synthetized (GenePharma, China), and a final concentration of $50 \mathrm{nM}$ was used for transient transfection. For overexpression of Inc-AC111653.1, full-length rat Inc-AC111653.1 cDNA was cloned into the pcDNA3.1 expression vector (GenePharma). Lipofectamine 3000 (Invitrogen) was used for transfection according to manufacturer's instructions (26).

\section{Western blotting}

Total proteins were extracted from cell lysates and separated by $10 \%$ SDS-polyacrylamide gel. Then, they were electroblotted to PVDF membranes (Beyotime, China). Membranes were incubated in rabbit polyclonal HYPM antibody (Novus Biologicals, China), followed by horseradish peroxidase-labeled goat anti-rabbit secondary antibody (Boster, China). Signals were revealed using ECL detection reagent (Beyotime). GAPDH served as control. Images were analyzed by Image-Pro Plus 6 software (Media Cybernetics, USA). The intensity of test protein bands was normalized to the GAPDH bands.

\section{Results}

\section{Gene mapping and differential expression genes}

The RNA quality and sequencing quality were guaranteed. Clean reads were obtained. The results of gene mapping to the rat genome is shown in Table 2. Known gene expression is shown in Tables 3 and 4.

On differential expression analysis, a total of 18,824 genes were included, of which there were 2643 differential genes between DRG test group and sham-operation group. By comparison of the DRG group with the sham-operation group, 1228 were up-regulated and 1415 down-regulated. On enrichment analysis of DEGs, up-regulated differential genes were attributed to $624 \mathrm{GO}$ terms and $50 \mathrm{KEGG}$ pathways; down-regulated differential genes were attributed to $424 \mathrm{GO}$ terms and $30 \mathrm{KEGG}$ pathways. DEGs were clustered into a heatmap (Figure 1).

\section{Known IncRNA, co-expression network, and target gene enrichment}

We found 69 neurite-associated known IncRNA genes linking to 866 target mRNA genes (Table 5). After the GO and KEGG enrichment information was presented at a $P$ value threshold $<0.1$, the 866 targets were enriched to $737 \mathrm{GO}$ terms and $40 \mathrm{KEGG}$ pathways. They were involved either in the downregulation of neurotransmitter 
Table 2. Mapping results of transcriptome to referenced genome.

\begin{tabular}{lccrr}
\hline Sample & Total Reads $(M)$ & Total Mapped $(M)$ & Multiple Mapped (M) & Uniquely Mapped (M) \\
\hline Sham 1 & 34,697 & $27,586(79.50 \%)$ & $7,890(22.74 \%)$ & $19,696(56.76 \%)$ \\
Sham 2 & 31,648 & $25,293(79.92 \%)$ & $7,034(22.23 \%)$ & $18,258(57.69 \%)$ \\
Sham 3 & 40,603 & $30,892(76.08 \%)$ & $9,391(23.13 \%)$ & $21,501(52.95 \%)$ \\
DRG1 & 24,133 & $14,275(59.15 \%)$ & $6,434(26.66 \%)$ & $7,840(32.49 \%)$ \\
DRG2 & 25,319 & $17,235(68.07 \%)$ & $7,139(28.20 \%)$ & $10,096(39.88 \%)$ \\
DRG3 & 41,835 & $30,454(72.80 \%)$ & $10,614(25.37 \%)$ & $19,841(47.43 \%)$ \\
\hline
\end{tabular}

DRG: dorsal root ganglia.

Table 3. Number and distribution of known gene expression.

\begin{tabular}{lcccccccrr}
\hline Sample & Genes & Min. & 1st Qu. & Median & Mean & 3rd Qu. & Max. & Sd. & Sum. \\
\hline Sham & 17856 & 0 & 0.83 & 4.75 & 151.65 & 14.99 & 482618 & 5987.02 & 2707906 \\
DRG & 17296 & 0 & 1.15 & 5.15 & 400.95 & 14.28 & 1828940 & 19976.57 & 6934761 \\
\hline
\end{tabular}

DRG: dorsal root ganglia.

Table 4. Number and percentage of known gene expression.

\begin{tabular}{lcccccc}
\hline Sample & $0-0.5$ & $>0.5-1$ & $>1-5$ & $>5-10$ & $>10-50$ & $>50$ \\
\hline Sham & $3634(20.35 \%)$ & $1184(6.63 \%)$ & $4322(24 \%)$ & $2764(15.48 \%)$ & $4488(25.13 \%)$ & $1464(0.08 \%)$ \\
DRG & $2859(16.53 \%)$ & $1175(6.79 \%)$ & $4504(26 \%)$ & $2996(17.32 \%)$ & $4416(25.53 \%)$ & $1346(0.08 \%)$ \\
\hline
\end{tabular}

DRG: dorsal root ganglia.

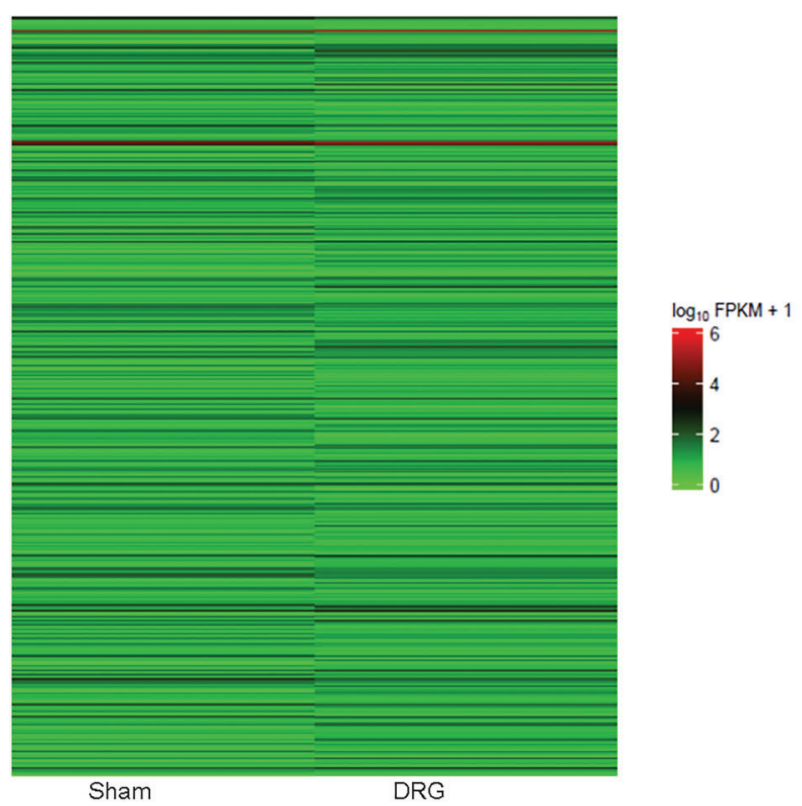

Figure 1. Genetic clustering of differential expression genes (threshold: fold-change $>2$ ). Red color represents high fold-change, green color represents low fold-change. Names were omitted due to many lines overlapping. DRG: dorsal root ganglia. status of neurons or in the upregulation of peripheral neuronal regeneration. The GO terms and KEGG pathways involved in the downregulation effects included, but were not limited to, synaptic vesicle exocytosis, neurotransmitter secretion, voltage-gated potassium channel activity, regulation of synaptic transmission, GABAergic synapse, response to pain, endocytosis, neuronal action potential, detection of mechanical stimulus involved in sensory perception of pain, neurotransmitter transport, the GABAergic synapse pathway, the cholinergic synapse pathway, the neuroactive ligand-receptor interaction pathway, the dopaminergic synapse pathway, and the synaptic vesicle cycle pathway. The GO terms and KEGG pathways involved in the upregulation effects included, but were not limited to, response to mechanical stimulus, regulation of cell growth, positive regulation of cell migration, positive regulation of ERK1 and ERK2 cascade, positive regulation of PI3K signaling, activation of MAPKK activity, cell differentiation, regulation of neuron projection regeneration, regulation of nerve growth factor receptor activity, peripheral nervous system axon regeneration, glial cell differentiation, the AMPK signaling pathway, the calcium signaling pathway, the PI3K-Akt signaling pathway, the glucose metabolism pathway, the MAPK signaling pathway, and the CGMP-PKG signaling pathway. 
Table 5. Known long non-coding RNA genes and their node degrees in Cytoscape co-expression network.

\begin{tabular}{lrlrlrl}
\hline nodes_label & nodes_degree & nodes_label & nodes_degree & nodes_label & nodes_degree \\
\hline ENSRNOG00000002734 & 47 & ENSRNOG00000052027 & 3 & ENSRNOG00000056599 & 4 \\
ENSRNOG00000003025 & 47 & ENSRNOG00000052373 & 1 & ENSRNOG00000056608 & 2 \\
ENSRNOG00000005811 & 1 & ENSRNOG00000052439 & 3 & ENSRNOG00000056656 & 1 \\
ENSRNOG00000006617 & 43 & ENSRNOG00000052563 & 37 & ENSRNOG00000056824 & 3 \\
ENSRNOG00000009373 & 1 & ENSRNOG00000052573 & 2 & ENSRNOG00000057161 & 12 \\
ENSRNOG00000011160 & 45 & ENSRNOG00000053160 & 2 & ENSRNOG00000057278 & 13 \\
ENSRNOG00000017974 & 4 & ENSRNOG00000053367 & 6 & ENSRNOG00000057291 & 1 \\
ENSRNOG00000019648 & 122 & ENSRNOG00000053827 & 1 & ENSRNOG00000057463 & 1 \\
ENSRNOG00000024799 & 55 & ENSRNOG00000054418 & 2 & ENSRNOG00000057991 & 1 \\
ENSRNOG00000031706 & 4 & ENSRNOG00000054489 & 1 & ENSRNOG00000058263 & 1 \\
ENSRNOG00000033581 & 88 & ENSRNOG00000054529 & 2 & ENSRNOG00000058571 & 12 \\
ENSRNOG00000043199 & 13 & ENSRNOG00000054533 & 5 & ENSRNOG00000058935 & 2 \\
ENSRNOG00000043866 & 5 & ENSRNOG00000054867 & 3 & ENSRNOG00000058944 & 3 \\
ENSRNOG00000046171 & 21 & ENSRNOG00000054897 & 3 & ENSRNOG00000059449 & 3 \\
ENSRNOG00000046774 & 3 & ENSRNOG00000054935 & 1 & ENSRNOG00000059660 & 1 \\
ENSRNOG00000047117 & 1 & ENSRNOG00000054984 & 1 & ENSRNOG00000060090 & 6 \\
ENSRNOG00000048929 & 31 & ENSRNOG00000055021 & 2 & ENSRNOG00000060430 & 2 \\
ENSRNOG00000049537 & 12 & ENSRNOG00000055067 & 1 & ENSRNOG00000060483 & 1 \\
ENSRNOG00000051356 & 2 & ENSRNOG00000055278 & 2 & ENSRNOG00000060700 & 1 \\
ENSRNOG00000051492 & 13 & ENSRNOG00000055939 & 42 & ENSRNOG00000060863 & 64 \\
ENSRNOG00000051664 & 29 & ENSRNOG00000056040 & 7 & ENSRNOG00000061151 & 2 \\
ENSRNOG00000051722 & 3 & ENSRNOG00000056054 & 5 & ENSRNOG00000061536 & 3 \\
ENSRNOG00000051924 & 3 & ENSRNOG00000056490 & 1 & ENSRNOG00000061622 & 1 \\
\hline & & & & &
\end{tabular}

The target gene from GO enrichment of known IncRNA apparently pointed to ENSRNOG00000006617 $(\mathrm{P}<0.05)$, thus we singled out the known IncRNA gene ENSRNOG00000006617 named rno-Cntnap2 (contactin associated protein-like 2). Through serial analyses of molecular network (Figure 2) and GO enrichment on gene rno-Cntnap2, we found 13 credible $\mathrm{GO}$ terms at $\mathrm{P}<0.05$ (Table 6).

According to the $13 \mathrm{GO}$ terms, rno-Cntnap2 had a putative gene function that is involved in the cell component of voltage-gated potassium channel complex on cell surface of brain neurites where it has an enzyme binding activity. Considering the condition of the current study, it was assumed that rno-Cntnap2 is involved in the cell component of voltage-gated potassium channel complex on cell surface of sciatic nerve neurites.

We reviewed the differential expression and co-expression network analysis of rno-Cntnap2 (ENSRNOG 00000006617) gene, which was down-regulated (20.34 and 3.94 for sham group vs DRG group, fold change -2.37 , $\mathrm{P}<0.001, \mathrm{Q}=0.0003$ ).

\section{Novel IncRNA, co-expression network, and target gene features}

We found 525 novel transcripts containing 26 novel IncRNAs referenced to rat IncRNA database (Table 7). We constructed the co-network of novel IncRNAs with
mRNAs, and only 4 IncRNAs were related to 21 mRNAs under the conditions of thresholds of $<0.05$ or 0.1 (Figure 3 ). The 4 IncRNAs were ENSRNOG00000055411, 000000 59555, 00000059564 and 00000057337 (Table 7).

We noticed that the transcript TCONS 00016823 included only one novel IncRNA gene, $\overline{A C} 111653.1$ (ENSRNOG00000057337), with a sense strand length of $828 \mathrm{nt}$. Gene AC111653.1 was null expressed in the sham-operation group and upregulated to 0.527889 in the DRG group. Gene AC111653.1 was correlated to the target gene ENSRNOG00000021452 named huntingtin interacting protein $M$ (rno-Hypm). The rno-Hypm gene $\mathrm{GO}$ annotations included the molecular function of DNA binding and protein heterodimerization activity, the biological process of chromatin silencing, and the cellular component of nuclear chromatin and nucleosome. Huntingtin is essential for neuron survival, and the lack of huntingtin synthesis may lead to Huntington's disease (27). Up-regulation of both AC111653.1 and rno-Hypm genes after sciatic nerve injury implies a rescue course that triggers the regeneration of injured neurons. However, the function of Hypm gene is not completely understood.

\section{Quantification of several genes}

For known IncRNAs detection, we selected rno-Cntnap2 IncRNA gene and three down-regulated gene representatives, Fam171b (ENSRNOG00000004783), Hebp2 


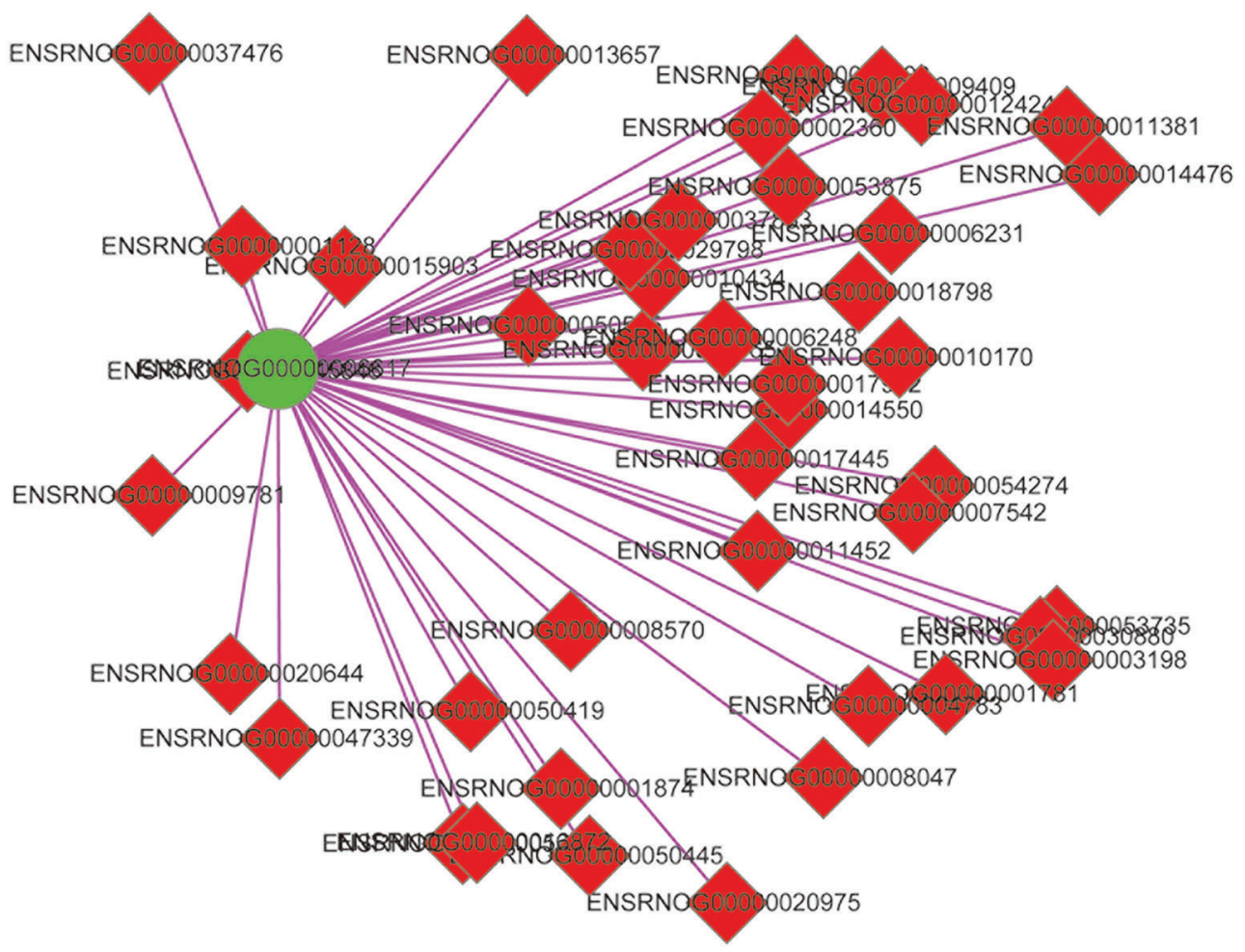

Figure 2. Co-expression network of gene rno-Cntnap2 (ENSRNOG00000006617).

Table 6. Gene Ontology (GO) terms of rno-Cntnap2 long non-coding RNA gene.

\begin{tabular}{lccc}
\hline Category & Term & Class & Gene_id \\
\hline GO:0071205 & protein localization to juxtaparanode region of axon & biological_process & ENSRNOG00000006617 \\
GO:0044224 & juxtaparanode region of axon & cellular_component & ENSRNOG00000006617 \\
GO:0030673 & axolemma & cellular_component & ENSRNOG00000006617 \\
GO:0008076 & voltage-gated potassium channel complex & cellular_component & ENSRNOG00000006617 \\
GO:0019899 & enzyme binding & molecular_function & ENSRNOG00000006617 \\
GO:0043204 & perikaryon & cellular_component & ENSRNOG00000006617 \\
GO:0031175 & neuron projection development & biological_process & ENSRNOG00000006617 \\
GO:0005769 & early endosome & cellular_component & ENSRNOG00000006617 \\
GO:0007420 & brain development & biological_process & ENSRNOG00000006617 \\
GO:0030424 & axon & cellular_component & ENSRNOG00000006617 \\
GO:0030425 & dendrite & cellular_component & ENSRNOG00000006617 \\
GO:0043025 & neuronal cell body & cellular_component & ENSRNOG00000006617 \\
GO:0009986 & cell surface & cellular_component & ENSRNOG00000006617 \\
\hline
\end{tabular}

(ENSRNOG00000053735), and Gde1 (ENSRNOG00000 050445) from the rno-Cntnap2 gene coexpression network (Figure 2). Real time qPCR was performed to quantify expression levels of the four genes. The quantification results are shown in Table 8. Expression levels of the four genes were down-regulated. This result was consistent with the sequencing outcomes.
For novel IncRNAs identification, we selected AC111653.1 gene (ENSRNOG00000057337) and rno-Hypm gene (ENSRNOG00000021452) from the AC111653.1 gene coexpression network (Figure 3 ). They had a correlation coefficient of 1 (significance $\mathrm{P}=0$ ). Up-regulation of both AC111653.1 and rno-Hypm genes after sciatic nerve injury may imply a rescue course that triggers the regeneration 
Table 7. Transcripts of 26 novel long non-coding RNA genes, of which 4 (in bold) are involved in co-expression network with target mRNA genes.

\begin{tabular}{lcccrc}
\hline Transcript & Length bp & gene_id & Transcript & Length bp & gene_id \\
\hline TCONS_00000233 & 509 & ENSRNOG00000058258 & TCONS_00016834 & 220 & ENSRNOG00000036434 \\
TCONS_00000914 & 1884 & ENSRNOG00000058637 & TCONS_00017920 & 149 & ENSRNOG00000058995 \\
TCONS_00001056 & 586 & ENSRNOG00000056324 & TCONS_00019224 & 1441 & ENSRNOG00000000809 \\
TCONS_00002131 & $\mathbf{8 7 9}$ & ENSRNOG00000055411 & TCONS_00021430 & 262 & ENSRNOG00000053319 \\
TCONS_00002609 & 194 & ENSRNOG00000060612 & TCONS_00021456 & 187 & ENSRNOG00000047611 \\
TCONS_00003909 & 1655 & ENSRNOG00000054067 & TCONS_00023970 & 2730 & ENSRNOG00000056448 \\
TCONS_00009651 & $\mathbf{5 9 3}$ & ENSRNOG00000059555 & TCONS_00028110 & 255 & ENSRNOG00000036492 \\
TCONS_00011039 & 350 & ENSRNOG00000061204 & TCONS_00028111 & 217 & ENSRNOG00000040358 \\
TCONS_00012047 & 330 & ENSRNOG00000035462 & TCONS_00028926 & 281 & ENSRNOG00000047126 \\
TCONS_00012411 & 134 & ENSRNOG00000052738 & TCONS_00033369 & 1000 & ENSRNOG00000057258 \\
TCONS_00014951 & $\mathbf{1 5 3}$ & ENSRNOG00000059564 & TCONS_00034793 & 312 & ENSRNOG00000032609 \\
TCONS_00015936 & 235 & ENSRNOG00000035501 & TCONS_00035961 & 1447 & ENSRNOG00000051245 \\
TCONS_00016823 & $\mathbf{8 2 8}$ & ENSRNOG00000057337 & TCONS_00036296 & 341 & ENSRNOG00000035579 \\
\hline
\end{tabular}
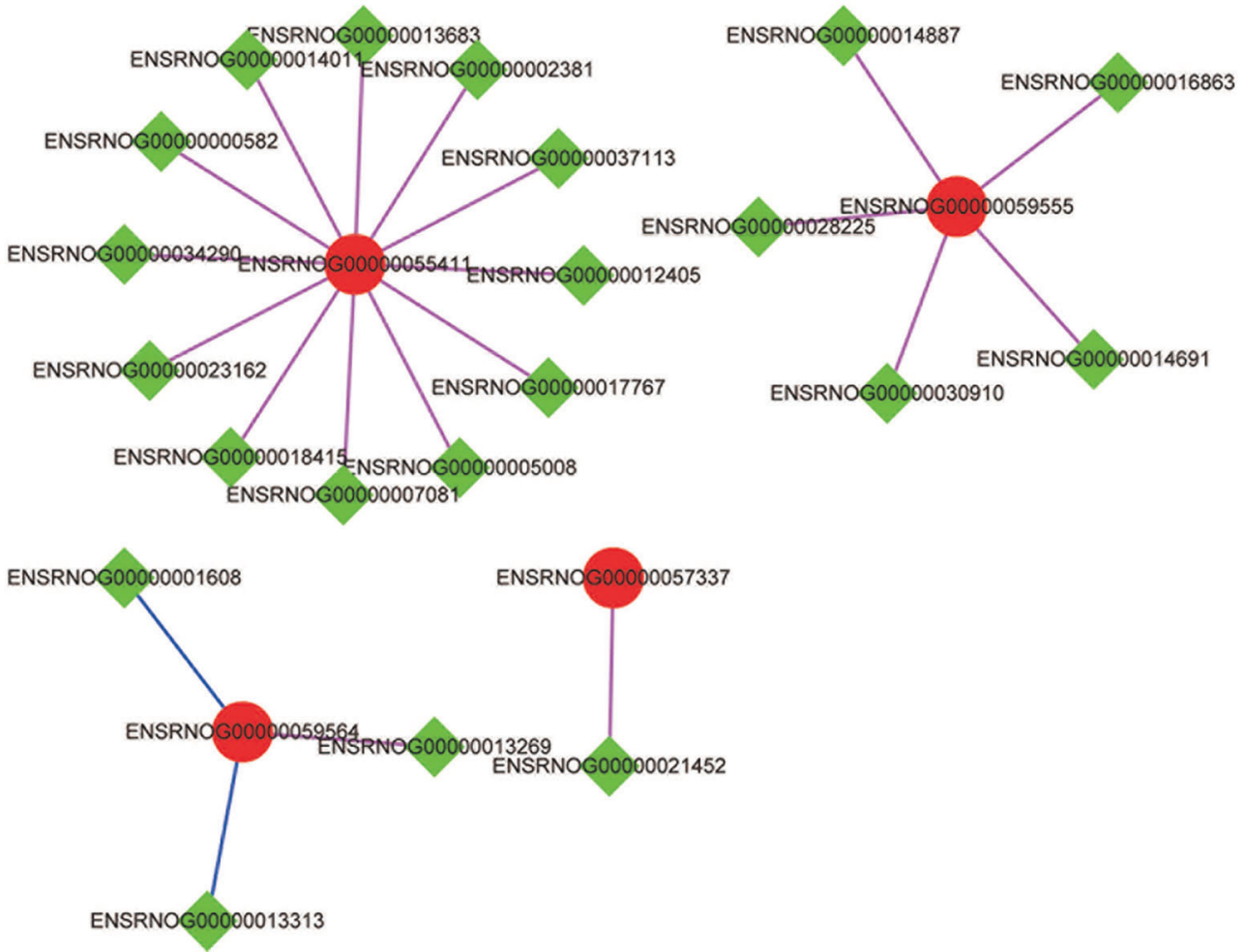

Figure 3. Co-expression network of novel long non-coding RNA (IncRNAs) with target mRNAs. The network comprises nodes and edges. Central round nodes are IncRNAs, green rectangle nodes are mRNAs. Purple edges indicate positive correlation and blue edges indicate negative correlation.

of injured neurons, thus the function of Hypm gene deserved to be studied. Real time qPCR was performed to quantify expression levels of the two genes (Table 8), which were up-regulated. This result was consistent with the sequencing outcomes.

\section{Cell assays}

To test the biological function of novel IncRNA AC111653.1 gene, we detected AC111653.1 and its target Hypm in primarily cultured Norway rat DRG cells in vitro. QPCR results are shown in Figure 4, and western blots in 
Table 8. Real time qPCR quantification of interest/GAPDH (interest DRG/Sham) gene expression levels.

\begin{tabular}{lccccc}
\hline & Sham & DRG1 & DRG2 & DRG3 & Sequencing (Sham/DRG) \\
\hline rno-Cntnap2-20 IncRNA & $0.0530(1.0000)$ & $0.0074(0.1411)$ & $0.0069(0.1310)$ & $0.0039(0.0736)$ & $20.3371 / 3.9422$ \\
rno-Fam171b & $0.0265(1.0000)$ & $0.0053(0.1993)$ & $0.0012(0.0446)$ & $0.0026(0.0981)$ & $19.7562 / 6.0137$ \\
rno-Hebp2 & $0.0417(1.0000)$ & $0.0112(0.2673)$ & $0.0145(0.3475)$ & $0.0082(0.1966)$ & $97.8422 / 22.9642$ \\
rno-Gde1 & $0.0128(1.0000)$ & $0.0097(0.7526)$ & $0.0042(0.3231)$ & $0.0065(0.5078)$ & $93.1178 / 36.7535$ \\
rno-AC111653.1 IncRNA & 0 & 0.08652 & 0.04228 & 0.03684 & $0 / 0.52789$ \\
rno-Hypm & 0 & 0.29365 & 0.1263 & 0.1046 & $0 / 2.6653$ \\
\hline
\end{tabular}

DRG: dorsal root ganglia. The numbers outside parenthesis indicate the gene expression ratio of interest to GAPDH and those inside indicate the gene expression ratio of interest DRG groups to the Sham group.

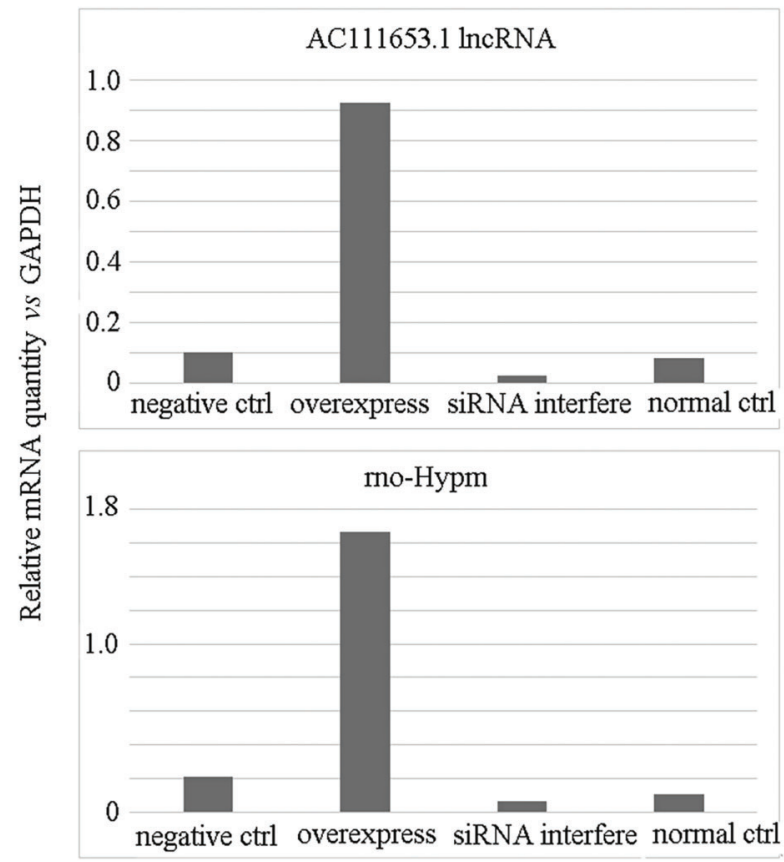

Figure 4. QPCR relative mRNA quantification. Upper panel shows rno-IncRNA AC111653.1 levels. Lower panel shows rno-Hypm mRNA levels in each group. Negative ctrl: pcDNA3.1 vector transfection; overexpress: pcDNA3.1-Inc-AC111653.1 transfection; siRNA interfere: siRNA-Inc-AC111653.1 transfection; normal ctrl: normal cells without treatment.

Figure 5. Novel IncRNA AC111653.1 was overexpressed after pcDNA3.1-Inc-AC111653.1 transfection. At the same time, its target hypm was also upregulated. Expression of AC111653.1 was reduced after siRNA transfection, and at the same time, its target hypm was downregulated. This suggested that novel IncRNA AC111653.1 was positively associated with hypm regulation in rats.

\section{Discussion}

In this study, we used a common sciatic nerve injury model to investigate gene expression conditions in rat

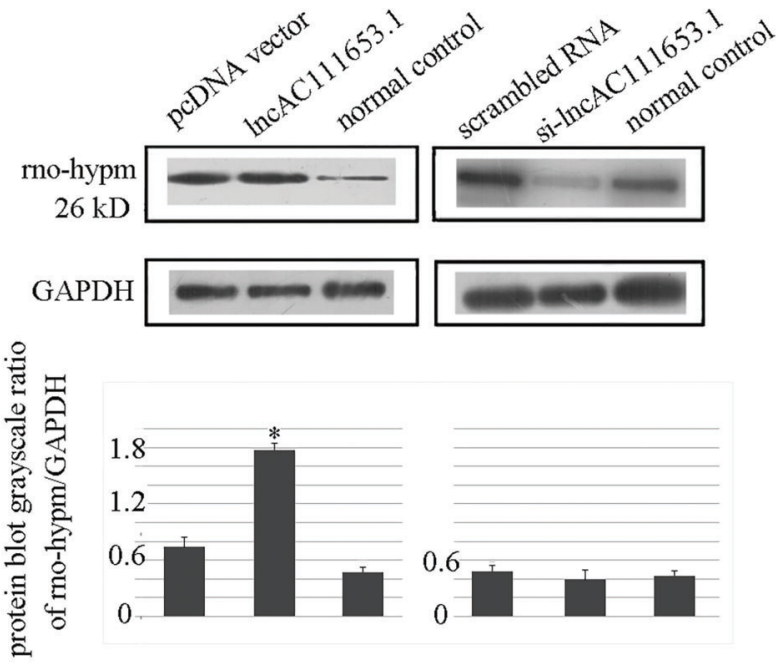

Figure 5. Western blot images. Cells were transfected with pcDNA3.1-Inc-AC111653.1 for overexpression and with siRNAInc-AC111653.1 for RNA interference. pcDNA3.1 vector and scrambled RNA served as negative controls. ${ }^{*} \mathrm{P}<0.05$ compared to the other groups (ANOVA).

DRGs using a high-throughput Illumina HiSeq2500 sequencing. In total, 86 known IncRNAs and 26 novel IncRNAs were altered during nerve regeneration. To understand the functions of the 86 known IncRNAs, we analyzed the molecular network including 866 co-expressed target genes. After sciatic nerve damage, the nerve systems switched from a neurotransmitter status to a neuronal regeneration status $(1,3,5)$.

Based on the GO and KEGG enrichment results, we found that the neurotransmitter status of neurons are deregulated by the molecular mechanisms linking to the deregulation of the neuroactive neurotransmitter secretion, transmission, and ligand-receptor interaction pathway, while the neuronal regeneration was activated through the molecular mechanisms linking to the positive regulation of cell migration, cell differentiation, cell growth, PI3K signaling, MAPK cascade activity, nerve growth factor receptor activity, and peripheral nerve regeneration. 
Glial cells migration, dedifferentiation, differentiation, proliferation, and growth play important roles in peripheral nerve regeneration (1-4). The results in this study showed the promotion of glial cell migration and growth by multiple signaling pathways. After sciatic nerve damage, local Schwann cells can shed off the myelin sheaths and transform to a neuroblast status, where their proliferation and migration capacities can help to sweep away myelin remnants and generate a conduit for the axonal pathfinding, and consequently form the beneficial conditions for neurite outgrowth (1-3). The same IncRNA-linked nerve regeneration mechanism is identified by Yu et al. (16) and Yao et al. (18).

We singled out the known rno-Cntnap2 IncRNA gene, thought to be involved in the cell component of voltagegated potassium channel complex on cell surface for the neurites of the sciatic nerve system. We speculated that sciatic injury might trigger a switch from a neurotransmitter status to a regeneration status of neurons. The gene rno-Cntnap2 may be involved in a neurotransmitter delivery process linking to the function of voltage-gated potassium channel complex. Thus, rno-Cntnap2 gene expression was down-regulated because a neurotransmitter status was ceased. The modulation of voltage-gated potassium channels by a IncRNA has been identified in DRG first-order sensory neurons in a spinal nerve ligation rat model (17). In this previous study, peripheral nerve injury increased a conserved IncRNA (Kcna2 antisense RNA) expression in injured DRG through activation of the transcription factor myeloid zinc finger protein 1. This increase of IncRNA

\section{References}

1. Reier PJ, Lane MA. Degeneration, regeneration, and plasticity in the nervous system. In: Conn PM (Editors), Neuroscience in Medicine, 3rd Edition. Totowa, NJ: Humana Press, 2008. p 691-727, doi: 10.1007/978-1-60327-455-5.

2. DeFrancesco-Lisowitz A, Lindborg JA, Niemi JP, Zigmond $\mathrm{RE}$. The neuroimmunology of degeneration and regeneration in the peripheral nervous system. Neuroscience 2015; 302: 174-203, doi: 10.1016/j.neuroscience.2014.09.027.

3. Rodríguez FJ, Valero-Cabré A, Navarro X. Regeneration and functional recovery following peripheral nerve injury. Drug Discovery Today Disease Models 2004; 1: 177-185, doi: 10.1016/j.ddmod.2004.09.008.

4. Angius $D$, Wang H, Spinner RJ, Gutierrez-Cotto $Y$, Yaszemski MJ, Windebank AJ. A systematic review of animal models used to study nerve regeneration in tissue-engineered scaffolds. Biomaterials 2012; 33: 8034-8039, doi: 10.1016/ j.biomaterials.2012.07.056

5. Vogelaar CF, Hoekman MF, Gispen WH, Burbach JP. Homeobox gene expression in adult dorsal root ganglia during sciatic nerve regeneration: is regeneration a recapitulation of development? Eur J Pharmacol 2003; 480: 233250, doi: 10.1016/j.ejphar.2003.08.110. downregulates the voltage-dependent potassium channel Kcna2 mRNA, consequently reducing total potassium current. The decrease of potassium current increases the neural excitability, namely neuropathy-induced sensitivity to mechanical stimuli in DRG neurons, resulting in neuropathic pain symptoms. The modulation of rno-Cntnap2 mRNA may also follow this molecular mechanism, though identification is required.

We further selected the transcript TCONS_00016823 containing only one novel IncRNA gene $\overline{A C} 111653.1$ (ENSRNOG0000005733). This IncRNA's upregulation improved the rno-Hypm gene expression, which promoted huntingtin synthesis regenerating sciatic nerves. We tested the biological function of novel IncRNA AC111653.1 in rat dorsal root ganglion cells. The overexpression of IncRNA AC111653.1 upregulated rno-Hypm gene substantially, indicating that this novel IncRNA is accurately associated with the huntingtin protein regulation.

The time-course factor should be considered a limitation because transcript levels vary depending on the time between the mechanic stimuli of nerve tying until the detection starts (16). Thus, time-dependent gene expression change and more testing on IncRNA functions should be done in the future. In addition, more annotations on genes should be investigated.

In conclusion, a total of 26 novel IncRNAs were found. Both down-regulated rno-Cntnap2 gene and up-regulated rno-Hypm gene were involved in neuropathic pain of DRGs after spared sciatic nerve injury, thus contributing to peripheral nerve regeneration via putative mechanisms.
6. Moore DL, Goldberg JL. Multiple transcription factor families regulate axon growth and regeneration. Dev Neurobiol 2011; 71: 1186-1211, doi: 10.1002/dneu.20934.

7. Allodi I, Udina E, Navarro X. Specificity of peripheral nerve regeneration: interactions at the axon level. Prog Neurobiol 2012; 98: 16-37, doi: 10.1016/j.pneurobio.2012.05.005.

8. Bali KK, Kuner R. Noncoding RNAs: key molecules in understanding and treating pain. Trends Mol Med 2014; 20: 437-448, doi: 10.1016/j.molmed.2014.05.006.

9. Jiang BC, Sun WX, He LN, Cao DL, Zhang ZJ, Gao YJ. Identification of IncRNA expression profile in the spinal cord of mice following spinal nerve ligation-induced neuropathic pain. Mol Pain 2015; 11: 43, doi: 10.1186/s12990-0150047-9.

10. Ponting CP, Oliver PL, Reik W. Evolution and functions of long noncoding RNAs. Cell 2009; 136: 629-641, doi: 10.1016/ j.cell.2009.02.006.

11. Derrien T, Johnson R, Bussotti G, Tanzer A, Djebali S, Tilgner $\mathrm{H}$, et al. The GENCODE $\mathrm{v} 7$ catalog of human long noncoding RNAs: analysis of their gene structure, evolution, and expression. Genome Res 2012; 22: 1775-1789, doi: 10.1101/gr.132159.111. 
12. Qureshi IA, Mehler MF. Long non-coding RNAs: novel targets for nervous system disease diagnosis and therapy. Neurotherapeutics 2013; 10: 632-646, doi: 10.1007/s13311013-0199-0.

13. Knauss JL, Sun T. Regulatory mechanisms of long noncoding RNAs in vertebrate central nervous system development and function. Neuroscience 2013; 235: 200-214, doi: 10.1016/ j.neuroscience.2013.01.022.

14. Wu P, Zuo X, Deng H, Liu X, Liu L, Ji A. Roles of long noncoding RNAs in brain development, functional diversification and neurodegenerative diseases. Brain Res Bull 2013; 97: 69-80, doi: 10.1016/j.brainresbull.2013.06.001.

15. Zuo L, Tan Y, Wang Z, Wang KS, Zhang X, Chen X, et al. Long noncoding RNAs in psychiatric disorders. Psychiatr Genet 2016; 26: 109-116, doi: 10.1097/YPG.000000000 0000129.

16. Yu B, Zhou S, Hu W, Qian T, Gao R, Ding G, et al. Altered long noncoding RNA expressions in dorsal root ganglion after rat sciatic nerve injury. Neurosci Lett 2013; 534: 117-122, doi: 10.1016/j.neulet.2012.12.014.

17. Zhao X, Tang Z, Zhang H, Atianjoh FE, Zhao JY, Liang L, et al. A long noncoding RNA contributes to neuropathic pain by silencing Kcna2 in primary afferent neurons. Nat Neurosci 2013; 16: 1024-1031, doi: 10.1038/nn.3438.

18. Yao C, Wang J, Zhang H, Zhou S, Qian T, Ding F, et al. Long non-coding RNA uc.217 regulates neurite outgrowth in dorsal root ganglion neurons following peripheral nerve injury. Eur J Neurosci 2015; 42: 1718-1725, doi: 10.1111/ ejn. 12966 .

19. Liu S, Zou L, Xie J, Xie W, Wen S, Xie Q, et al. LncRNA NONRATT021972 siRNA regulates neuropathic pain behaviors in type 2 diabetic rats through the P2X7receptor in dorsal root ganglia. Mol Brain 2016; 9: 44, doi: 10.1186/ s13041-016-0226-2.
20. Peng H, Zou L, Xie J, Wu H, Wu B, Zhu G, et al. LncRNA NONRATT021972 siRNA decreases diabetic neuropathic pain mediated by the $\mathrm{P} 2 \mathrm{X} 3$ receptor in dorsal root ganglia. Mol Neurobiol 2017; 54: 511-523, doi: 10.1007/s12035-0159632-1.

21. Wang S, Xu H, Zou L, Xie J, Wu H, Wu B, et al. LncRNA uc. $48+$ is involved in diabetic neuropathic pain mediated by the P2X3 receptor in the dorsal root ganglia. Purinergic Signal 2016; 12: 139-148, doi: 10.1007/s11302-015-9488-x.

22. North RA. Molecular physiology of P2X receptors. Physiol Rev 2002; 82: 1013-1067, doi: 10.1152/physrev.00015.2002.

23. Burnstock G. P2X receptors in sensory neurones. $\mathrm{Br} \mathrm{J}$ Anaesth 2000; 84: 476-488, doi: 10.1093/oxfordjournals. bja.a013473

24. Smith SM, Murray DW. An overview of microRNA methods: expression profiling and target identification. In: Espina V (Editor). Molecular Profiling: Methods and Protocols, Methods in Molecular Biology. Germany, Berlin: Springer Press, 2012. p 119-138, doi: 10.1007/978-1-60327-216-2.

25. Haller I, Lirk P, Keller C, Wang GK, Gerner P, Klimaschewski L. Differential neurotoxicity of tricyclic antidepressants and novel derivatives in vitro in a dorsal root ganglion cell culture model. Eur J Anaesthesiol 2007; 24: 702-708, doi: 10.1017/ S0265021507000154.

26. Ye C, Shen Z, Wang B, Li Y, Li T, Yang Y, et al. A novel long non-coding RNA Inc-GNAT1-1 is low expressed in colorectal cancer and acts as a tumor suppressor through regulating RKIP-NF-кB-Snail circuit. J Exp Clin Cancer Res 2016; 35 : 187, doi: 10.1186/s13046-016-0467-z.

27. Butland SL, Sanders SS, Schmidt ME, Riechers SP, Lin DT, Martin DD, et al. The palmitoyl acyltransferase HIP14 shares a high proportion of interactors with huntingtin: implications for a role in the pathogenesis of Huntington's disease. Hum Mol Genet 2014; 23: 4142-4160, doi: 10.1093/hmg/ddu137. 